\title{
ARTICLE Minocycline protects against myocardial ischemia/reperfusion injury in rats by upregulating MCPIP1 to inhibit NF-кB activation
}

\author{
Quan $\mathrm{Yi}^{1}$, Fang-hui Tan ${ }^{1}$, Jia-an Tan ${ }^{1}$, Xiu-hui Chen ${ }^{1}$, Qing Xiao ${ }^{1}$, Ying-hua Liu ${ }^{1}$, Gui-ping Zhang ${ }^{1}$ and Jian-dong Luo ${ }^{1,2}$
}

\begin{abstract}
Minocycline is a tetracycline antibiotic and has been shown to play a protective role in cerebral and myocardial ischemia/ reperfusion (I/R). However, the underlying mechanism remains unclear. Herein, we investigated whether monocyte chemotactic protein-induced protein-1 (MCPIP1), a negative regulator of inflammation, was involved in the minocycline-induced cardioprotection in myocardial I/R in vivo and in vitro models. Myocardial ischemia was induced in rats by left anterior descending coronary artery occlusion for $1 \mathrm{~h}$ and followed by $48 \mathrm{~h}$ reperfusion. Minocycline was administered prior to ischemia (45 mg/kg, ip, BID, for $1 \mathrm{~d}$ ) and over the course of reperfusion $(22.5 \mathrm{mg} / \mathrm{kg}$, ip, BID, for $2 \mathrm{~d}$ ). Cardiac function and infarct sizes were assessed. Administration of minocycline significantly decreased the infarct size, alleviated myocardial cell damage, elevated left ventricle ejection fraction, and left ventricle fractional shortening following I/R injury along with significantly decreased pro-inflammatory cytokine IL-1 $\beta$ and monocyte chemoattractant protein-1 (MCP-1) levels in heart tissue. H9c2 cardiomyocytes were subjected to oxygen glucose deprivation (OGD) followed by reoxygenation (OGD/R). Pretreatment with minocycline $(1-50 \mu \mathrm{mol} / \mathrm{L})$ dosedependently increased the cell viability and inhibited OGD/R-induced expression of MCP-1 and IL-6. Furthermore, minocycline dose-dependently inhibited nuclear translocation of NF-KB p65 in H9c2 cells subjected to OGD/R. In both the in vivo and in vitro models, minocycline significantly increased MCPIP1 protein expression; knockdown of MCPIP1 with siRNA in H9c2 cells abolished all the protective effects of minocycline against OGD/R-induced injury. Our results demonstrate that minocycline alleviates myocardial I/R injury via upregulating MCPIP1, then subsequently inhibiting NF-kB activation and pro-inflammatory cytokine secretion.
\end{abstract}

Keywords: myocardial ischemia and reperfusion; H9c2 cardiomyocytes; oxygen glucose deprivation; minocycline; cytokine; NF-kB; monocyte chemotactic protein-induced protein-1

Acta Pharmacologica Sinica (2019) 40:1019-1028; https://doi.org/10.1038/s41401-019-0214-z

\section{INTRODUCTION}

Coronary artery disease (CAD) is one of the most common cardiac diseases and a major cause of morbidity and mortality worldwide. Myocardial tissue injury in acute myocardial infarction arises from arterial occlusion leading to ischemia and subsequent restoration of blood flow, which can inadvertently result in reperfusion injury [1]. Thus, myocardial ischemia/ reperfusion (I/R) injury is a two-hit process with many underlying pathological mechanisms, including robust inflammatory responses $[2,3]$. While the mortality rate associated with CAD has steadily declined in the past few decades due to the identification of therapeutic agents that mostly targeting the ischemic stage, CAD remains a leading cause of death in people older than 35 years [4]. Therefore, a deeper understanding of the pathological mechanisms of myocardial I/R injury is necessary to identify agents that are more effective, including those that target the second stage of injury (i.e., reperfusion).

Myocardial I/R injury triggers oxidative stress and is associated with the production of pro-inflammatory cytokines, including interleukin (IL)-1, IL-6, IL-8, and tumor necrosis factor-alpha (TNF-a).
Monocyte chemotactic protein-induced protein-1 (MCPIP1, also known as $\mathrm{ZC} 3 \mathrm{H} 12 \mathrm{~A}$ ) is an inducible protein expressed on monocytes upon treatment with monocyte chemoattractant protein-1 (MCP-1) [5] that was previously identified as a negative regulator of inflammation [6]. Specifically, MCPIP1 inhibits the production of MCP-1, IL-1 $\beta, I L-6$, and TNF- $\alpha$ via inhibition of the JNK and NF-KB signaling pathways $[7,8]$. Expression of MCPIP1 is elevated in the ventricular myocardium of patients with ischemic heart failure, and MCPIP1 attenuates post-infarct cardiac remodeling and dysfunction [9]. However, the relevance of this role of MCPIP1 in the pathogenesis of myocardial I/R injury is unknown. Minocycline, a member of the family of broad-spectrum bacteriostatic tetracycline antibiotics, was recently shown to possess anti-inflammatory properties [10] separate from its antimicrobial function and has been used for the treatment of diseases such as urethritis and rheumatoid arthritis [11]. Experimentally, minocycline has also been shown to exhibit neuroprotective and cardioprotective functions in animal models of cerebral $\mathrm{I} / \mathrm{R}$ or myocardial $\mathrm{I} / \mathrm{R}$ injury $[12,13]$. Recently, minocycline was found to mediate neuroprotection during cerebral ischemia via MCPIP1 [14]. However, the potential role of this

\footnotetext{
${ }^{1}$ Department of Pharmacology, School of Pharmaceutical Sciences, Guangzhou Medical University, Guangzhou 510182, China and ${ }^{2}$ Guangzhou Institute of Cardiovascular Disease, Guangzhou Key Laboratory of Cardiovascular Disease, Second Affiliated Hospital, Guangzhou Medical University, Guangzhou 510260, China Correspondence: Jian-dong Luo (jiandongluo@hotmail.com)
}

Received: 5 November 2018 Accepted: 16 January 2019

Published online: 21 February 2019 
minocycline-mediated cardioprotective mechanism in myocardial $\mathrm{l} / \mathrm{R}$ injury remains unknown.

The present study sought to investigate whether MCPIP1 is involved in the cardioprotective effects of minocycline in an animal model of myocardial I/R injury and to elucidate whether the anti-inflammatory potency of MCPIP1 contributes to minocycline-induced myocardial protection in vitro under oxygen glucose deprivation (OGD)/reoxygenation (OGD/R)-induced injury in $\mathrm{H} 9 \mathrm{c} 2$ cells.

\section{MATERIALS AND METHODS}

Animals

All animal experiments were performed in accordance with the Guidelines for the Care and Use of Laboratory Animals published by the U.S. National Institute of Health. The Institutional Animal Care and Use Committee of Guangzhou Medical University approved the study protocols.

Male Sprague-Dawley rats (age, 7-8 weeks; weight, 250-280 g) were obtained from the Medical Experimental Animal Center of Guangdong Province (Guangzhou, China). The animals (4/cage) were acclimated for two weeks prior to initiating experiments with free access to standard rat chow and water. The rats were randomly divided into three groups: (i) Sham (without LAD ligation, $n=6$ ); (ii) I/R model (occlusion and release of left anterior descending coronary artery [LAD] with the same volume of saline alone; $n=6$ ); and (iii) I/R + Mino (I/R plus minocycline treatment; $n=8$ ). Twelve hours prior to ischemia induction, the rats were injected intraperitoneally with $45 \mathrm{mg} / \mathrm{kg}$ minocycline hydrochloride (Aladdin, Shanghai, China) twice daily on the first day; thereafter, the animals were injected twice a day at a dose of $22.5 \mathrm{mg} / \mathrm{kg}$ for the subsequent two days during the course of reperfusion [12]. The experiments were completed at the end of the 48-h reperfusion period. Animals were euthanized after the completion of echocardiography.

\section{Myocardial I/R surgery}

Myocardial I/R surgery was performed as previously described [15]. Briefly, LAD was temporarily occluded and then released. The rats were anesthetized with pentobarbital $(30 \mathrm{mg} / \mathrm{kg}$; ip), and the LAD was ligated using a 5-0 silk suture (Ningbo Medical Needle Co., Ltd., Ningbo, China). The electrocardiogram (Fujifilm Visual Sonics, Inc., Toronto, ON, Canada) was monitored for changes in the ST-T segment resulting from tightening or loosening of the ligature. Following $60 \mathrm{~min}$ of ischemia, the myocardium was reperfused by restoring coronary circulation for $48 \mathrm{~h}$. The sham control group underwent similar procedures without LAD ligation.

\section{Echocardiography}

Transthoracic echocardiography was performed with a Visual Sonics instrument (Vevo 2100; Visual Sonics Inc., Toronto, ON, Canada) equipped with a $25 \mathrm{MHz}$ imaging transducer using previously published procedures [16]. Briefly, the rats were anaesthetized with $2 \%$ isoflurane gas with an inflow rate of $0.5-1.5 \mathrm{~mL} / \mathrm{min}$ during echocardiography measurements. The left ventricle (LV) was analyzed through the parasternal long- and short-axis views. The body temperature was maintained at $37^{\circ} \mathrm{C}$, the respiratory rate (RR) was monitored, and the heart rate (HR) was maintained at $\sim 350$ beats $/ \mathrm{min}$. After measurements, cardiac output values such as the left ventricle ejection fraction (LVEF), left ventricle fractional shortening (LVFS), and other parameters were calculated according to Vevo 2100 guidelines.

\section{Histopathological staining}

Heart histological examination was performed as previously described [17]. Briefly, after echocardiography assessment, the heart was quickly removed, and the left ventricle was excised, washed with ice-cold phosphate buffer saline (PBS), and fixed in $10 \%$ buffered formalin. Samples were dehydrated in graded ethanol, cleared with xylene, embedded in paraffin and cut into 10 pieces $2 \mathrm{~mm}$ below the ligation line, followed by hematoxylin and eosin (H\&E) staining of each $5-\mu \mathrm{m}$ section. Slides were imaged under a light microscope (Leica, Germany).

\section{Determination of infarct size}

At the end of $48 \mathrm{~h}$ of reperfusion, the LAD was re-ligated, and Evans blue dye was infused intravenously through the superior vena cava to determine the non-ischemic zone (area not at risk, ANAR). Then, the heart was removed and washed with saline, and the non-myocardial tissues, such as blood vessels and fat, were removed. The LV was sliced into parallel 2-mm-thick myocardial sections along the coronary sulcus from the apex to the floor of the heart. Next, the hearts were sliced transversely into five slices, which were incubated in $1.0 \%$ triphenyltetrazolium chloride (TTC, Sigma-Aldrich) solution at $37^{\circ} \mathrm{C}$ for $10 \mathrm{~min}$, to identify the viable and infarcted areas (I) in the ischemic myocardium (area at risk, AAR). Each slice was weighed and photographed, and each area was measured by Image Pro Plus 5.0 (Media Cybernetics Inc., Rockville, MD, USA). The volume of all areas was estimated with the following formula: volume of area $=(\mathrm{A} 1 \times \mathrm{Wt} 1)+(\mathrm{A} 2 \times \mathrm{Wt} 2)+$ $(\mathrm{A} 3 \times \mathrm{Wt} 3)+(\mathrm{A} 4 \times \mathrm{Wt} 4)+(\mathrm{A} 5 \times \mathrm{Wt} 5)$, where each $\mathrm{A}$ is the percentage of the area of each respective section and each $\mathrm{Wt}$ is the weight of each respective section. Viable non-ischemic myocardium stained blue, the ischemic area stained red, and the infarcted area was stained pale [18].

\section{Measurement of inflammatory cytokines}

Heart tissue homogenates were prepared as previously described [19]. Briefly, after I/R treatment with or without minocycline for the indicated times, the animal was euthanized, the circulatory system was flushed with $40 \mathrm{~mL}$ of $4{ }^{\circ} \mathrm{C}$ PBS injected through the left ventricle and drained from the left atrium, and the heart was collected. Proteins were extracted with mammalian cell/tissue lysis buffer (Cell Signaling Technology, Beverly, MA, USA), and protein content was quantified by the BCA protein assay (Pierce). The levels of MCP- 1 and IL-1 $\beta$ were assessed using the Bio-plex Pro ${ }^{T M}$ reagent kit (Cat\#171-304070 M) following the manufacturer's instructions. Data acquisition and analysis were conducted using the Bio-Plex 200 system.

\section{Cell culture}

The $\mathrm{H} 9 \mathrm{c} 2$ cell line, a subclone of the original clonal cell line derived from embryonic rat heart tissue, was obtained from American Type Culture Collection (ATCC, Rockville, MD, USA). The cells were cultured and subjected to OGD as described previously [20]. Briefly, the cells were cultured in Dulbecco's modified Eagle's medium (DMEM; Gibco, Logan, UT, USA) supplemented with $10 \%$ fetal bovine serum (FBS; Gibco, Logan, UT, USA) at $37^{\circ} \mathrm{C}$ under an atmosphere of $5 \% \mathrm{CO}_{2}$ and $95 \%$ air. Minocycline $(1-50 \mu \mathrm{M})$ (Sigma-Aldrich, St. Louis, MO, USA) was added to the culture medium $2 \mathrm{~h}$ prior to OGD treatment. For OGD experiments, the cells were rinsed twice with serum-free, glucose-free and sodium pyruvate-free DMEM (Cat\#D5030, Sigma-Aldrich) and were then placed in a $37^{\circ} \mathrm{C}$ incubator at $94 \% \mathrm{~N}_{2}, 5 \% \mathrm{CO}_{2}$ and $1 \% \mathrm{O}_{2}$ for $6 \mathrm{~h}$. The cells were then reoxygenated by return to normal feeding medium and incubated under normal conditions at $37^{\circ} \mathrm{C} 5 \% \mathrm{CO}_{2}$ and $95 \%$ air for an additional 1-6 h. The cells were then harvested to determine the extent of cell damage. Control cell cultures not deprived of oxygen and glucose were incubated under normal conditions.

MTS cell viability assay and lactate dehydrogenase activity assay $\mathrm{H} 9 \mathrm{c} 2$ cells were seeded in 96-well plates (Corning) at a density of $5 \times 10^{3}$ cells per well with $100 \mu \mathrm{L}$ of medium containing $10 \%$ FBS and incubated at $37{ }^{\circ} \mathrm{C}$ with $5 \% \mathrm{CO}_{2}$ for $24 \mathrm{~h}$. The cells were 
Table 1. The sequences for qPCR primers and siRNA oligonucleotides

\begin{tabular}{ll}
\hline Gene & Sequence \\
\hline MCPIP1 & F: 5'-CCAGGACTCCAGTCAAGGACAA-3'; \\
R: 5'-TGGCTCAGCCTCTAGGGACA-3'; & F: 5'-GGCGTGTTCATCCGTTCTC-3'; \\
R: 5'-CTTCAGCGTCTCGTGTGTTTCT-3'; & F: 5'-ATTGTATGAACAGCGATGATGCAC-3'; \\
IL-6 & R: 5'-CCAGGTAGAAACGGAACTCCAGA-3'; \\
MCP-1 & F: 5'-CTATGCAGGTCTCTGTCACGCTTC-3'; \\
& R: 5'-CAGCCGACTCATTGGGATCA-3'; \\
GAPDH & F: 5'-ACTCACTCAAGATTGTCAGCAATG-3'; \\
RiRNA oligonucleotides & R: 5'-TCATGAGCCCTTCCACAATGC-3' \\
MCPIP1-1 & 5'-UGAAAUGGCCAGUACCGAUGGCUUG-3' \\
MCPIP1-2 & 5'-UUCUUACGCAGGAAGUUGUCCAGGC-3' \\
MCPIP1-3 & 5'-AAGAAAGGAUCUCAGCGGCCAGCUG-3' \\
Control & Cat\#12935400 \\
\hline F forward sequence, R reverse sequence
\end{tabular}

$\mathrm{F}$ forward sequence, $\mathrm{R}$ reverse sequence

treated with varying concentrations of minocycline for $2 \mathrm{~h}$ and then subjected to OGD/R treatment for different time points as indicated. Similarly, another group of cells was transfected with siMCPIP1 $(20 \mathrm{nM})$ prior to OGD/R treatment. Cell proliferation was determined by using the CellTiter $96^{\circ}$ Aqueous One Solution Cell Proliferation Assay MTS (3-[4,5-dimethylthiazol2-yl]-5-[3-carboxymethoxyphenyl]-2-[4-sulfophenyl]-2H-tetrazolium, inner salt) kit (Cat\#G3582, Promega, Madison, WI, USA), and the absorbance at $490 \mathrm{~nm}$ was recorded using a 96-well plate reader (Bio-Rad, USA). Cell viability was calculated as follows: MTS metabolic rates $(\%)=\mathrm{A} 490$ in experimental well/ A490 in control well. Lactate dehydrogenase (LDH) activity was measured using a standard assay (Jiancheng, Nanjing, China) following the manufacturer's directions.

Cell apoptosis assay

Hoechst 33342 and propidium iodide (PI) double staining were used to detect cell apoptosis and necrosis. At the end of OGD treatment, $10 \mu \mathrm{g} / \mathrm{mL}$ Hoechst 33342 reagent was added to cells and incubated for $10 \mathrm{~min}$ in the dark at $37^{\circ} \mathrm{C}$, followed by the addition of $15 \mu \mathrm{g} / \mathrm{mL} \mathrm{PI}$ and incubation for $30 \mathrm{~min}$ in the dark at $4{ }^{\circ} \mathrm{C}$. After washing with PBS twice, fluorescence was imaged at $\times 40$ magnification. Apoptotic cells stained by Hoechst 33342 showed bright blue nuclear condensation and fragmentation, and necrotic cells displayed red fluorescence as stained by PI. Images were captured randomly using an Olympus digital camera (Olympus, Japan). For each sample, all cells with Hoechst-positive and Pl-positive cells were counted, and a representative image from at least 10 randomly chosen non-overlapping fields of view was used.

Quantitative real-time PCR

Total RNA was isolated using RNAiso Plus reagent (Takara Biotechnology Dalian Co., Ltd.). After removal of genomic DNA using the PrimeScript ${ }^{\mathrm{TM}}$ RT reagent Kit with gDNA Eraser (Takara), $1.0 \mu \mathrm{g}$ of total RNA from $\mathrm{H} 9 \mathrm{c} 2$ cells or the left ventricle of rat heart tissue was reverse-transcribed to cDNA using a commercially available kit (Takara). qRT-PCR was performed with an ABI-7500 iCycler Thermal Cycler (Applied Biosystems, Carlsbad, CA, USA) using SYBR Premix Ex Taq ${ }^{\mathrm{TM}}$ ॥| (Takara). Forty cycles were conducted as follows: $95^{\circ} \mathrm{C}$ for $5 \mathrm{~s}, 60^{\circ} \mathrm{C}$ for $30 \mathrm{~s}$, proceeded by $95^{\circ} \mathrm{C}$ for $30 \mathrm{~s}$ for polymerase activation. Quantification was performed by the delta cycle time method, with rat GAPDH used for normalization. Rat gene-specific primer sequences (Takara) are provided in Table 1.

Cell transfection

MCPIP1 siRNA (Cat\#1330003) and the negative control siRNA (Cat\#12935400) were mixed with Lipofectamine RNAiMAX Reagent (Cat\#13778150, Life Technologies, Inc., USA) for transfection according to the manufacturer's instructions. See Table 1 for oligonucleotide sequences. Briefly, $\mathrm{H} 9 \mathrm{c} 2$ cells were plated in $35-\mathrm{mm}$ dishes until reaching $50 \%$ confluence. The cells were starved and rinsed in serum-free medium, and Lipofectamine RNAiMAX reagent and siRNA were mixed and incubated for $5 \mathrm{~min}$ at room temperature before addition to the cells and incubation for $6 \mathrm{~h}$. After replacement with fresh medium, the cells were continuously cultured for another $30 \mathrm{~h}$. Next, the culture medium was replaced, and the cells were treated with or without minocycline $(50 \mu \mathrm{M})$ for $2 \mathrm{~h}$ prior to OGD/R treatment as described above. The transfection efficiency was determined by qPCR and Western blot analysis.

Western blot analysis

Immunoblot was performed as previously described [21]. Briefly, harvested $\mathrm{H} 9 \mathrm{C} 2$ cells or the border zone of an infarcted heart was separated and homogenized in lysis buffer (Cell Signaling Technology, USA) containing protease inhibitor cocktail (Cat\#14001, Bimake, Houston, TX, USA) and phosphatase inhibitor cocktail (Cat\#15001, Bimake, Houston, TX, USA) if phosphorylated proteins were detected. The samples were subsequently sonicated on ice, and the tissue lysates were boiled for $5 \mathrm{~min}$, followed by centrifugation at $12,000 \times g$ for $10 \mathrm{~min}$. The supernatants were collected, and protein content was analyzed using a BCA protein assay kit (Pierce). Nuclear and cytoplasmic proteins were extracted with a Nuclear and Cytoplasmic Extraction Kit (Keygentec, Nanjing, China). Equal amounts of cytoplasmic or nuclear protein $(30 \mu \mathrm{g})$ were separated on SDS polyacrylamide gels $(10 \%)$ and transferred to polyvinylidene difluoride (PVDF) membranes (Roche, Switzerland). The membranes were then blocked for $1 \mathrm{~h}$ at room temperature in TBS containing $0.1 \%$ Tween 20 (TTBS) and 5\% milk. Subsequently, the membranes were incubated overnight at $4{ }^{\circ} \mathrm{C}$ with appropriate primary antibodies in TTBS, followed by incubation with appropriate HRP-conjugated secondary antibodies. The protein-antibody complexes were visualized using LumiGLO $^{\circledR}$ chemiluminescent substrate (Cat\#7003, CST) and exposed to Kodak medical X-ray film (Kodak, Rochester, NY, USA). Data were quantified by specific molecular band intensity using Quantity One software (Bio-Rad) and normalized against GAPDH or Lamin B1. Anti-MCPIP1 (1:1000 dilution, Cat\#515275) was obtained from Santa Cruz Biotechnology (Santa Cruz, CA, USA), while anti-p-65 (1:1000 dilution, Cat\#3033, Cat\#8242) and anti-cleaved caspase 3 (1:1000 dilution, Cat\#9664) were obtained from Cell Signaling Technology (CST, Danvers, MA, USA).

\section{Confocal immunofluorescence microscopy}

The nuclear translocation of NF-кB p-65 was detected with laser confocal immunofluorescence microscopy. Briefly, H9c2 cells were fixed with $4 \%$ paraformaldehyde, permeabilized with $0.1 \%$ Triton-X-100, and incubated with a blocking buffer to prevent non-specific binding. Samples were then immunostained with an anti-NF-kB antibody directed against the p65 subunit overnight at $4{ }^{\circ} \mathrm{C}$, followed by incubation with DyLight ${ }^{\circ}$ 488-conjugated secondary antibody (Cat\#96899, Abcam, Cambridge, MA, USA) for $1 \mathrm{~h}$ in the dark. Nuclei were stained with 4',6-diamidino-2phenylindole (DAPI, Cat\#8961, CST, Danvers, MA, USA). After extensive washing with $\mathrm{PBS}$, the cells were imaged with a laser confocal fluorescence microscope (Alver 4.30, Nikon) at $\times 60$ magnification. 


\section{Statistical analysis}

Data were analyzed using SPSS17.0 (IBM Analytics, Chicago, IL, USA) and expressed as the mean \pm SEM. Statistical analyses were performed using a two-tailed unpaired Student's $t$ test for twogroup comparisons. Multiple comparisons were evaluated by oneway ANOVA followed by the specified post hoc test where appropriate. $P<0.05$ was considered significant.

\section{RESULTS}

Minocycline protects cardiomyocytes against I/R injury in vivo and OGD/R-mediated I/R injury in vitro

Previous studies have revealed a protective role for minocycline in myocardial I/R $[22,23]$. Herein, we examined the effect of minocycline treatment on myocardial $\mathrm{I} / \mathrm{R}$ infarction in vivo as described in the Methods section. The heart infarct size was evaluated using Evans blue dye-TTC double staining $48 \mathrm{~h}$ after reperfusion. The results showed that minocycline treatment did not alter the non-ischemic myocardium blue staining areas (i.e., the area not at risk, ANAR), and the ratio of the ischemic myocardium (AAR) to the total LV was not different in either group, indicating consistent I/R procedures; however, the I (infarcted areas) /LV and I/AAR ratios were significantly lower in the minocycline-treated rats (Fig. 1a; $17.84 \pm 3.12 \%$ vs $11.41 \pm$ $3.88 \% ; P<0.05$ and $33.66 \pm 4.31 \%$ vs $20.97 \pm 3.56 \% ; P<0.05)$. Furthermore, Evans blue dye-TTC double staining showed smaller infarct myocardium pale staining in the minocycline-treated rats. H\&E staining of the LV showed that in the I/R group, myocardial tissue was whiter below the ligation line, with increased leukocyte infiltration, irregular myocardial fibers, partial rupture, and obvious erythrocyte leakage, accompanied by granular and fatty degeneration of myocardial cells (Fig. $1 \mathrm{~b}$ arrow). By contrast, in the sham group, the myocardial fibers were arranged in a regular pattern, and structure was clear. Minocycline treatment significantly alleviated myocardial cell damage, accompanied by unbroken myocardial muscle fibers, ordered myocardial cells, reduced erythrocyte leakage and leukocyte infiltration, and no granular degeneration and lipoid degeneration. Rat cardiac function was

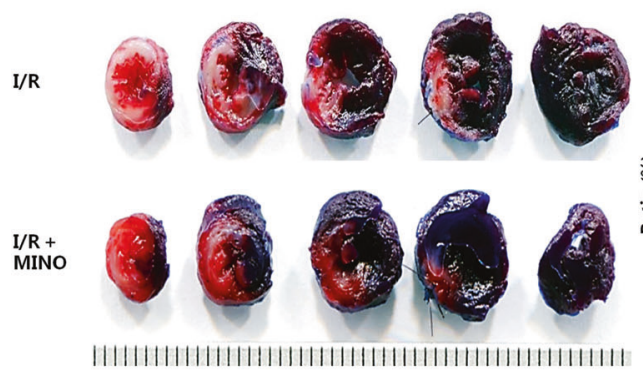

Sham
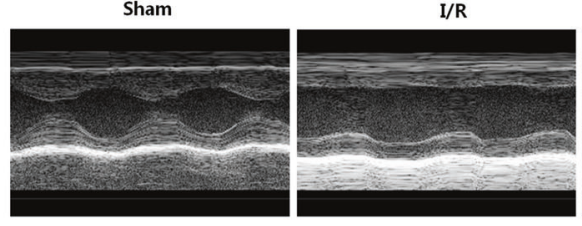

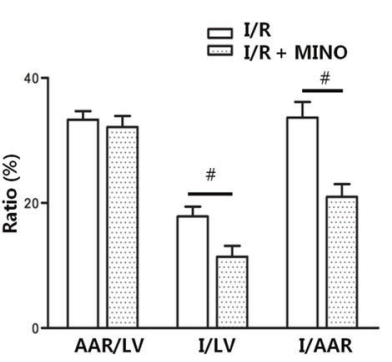

$\mathrm{I} / \mathrm{R}+\mathrm{MINO}$

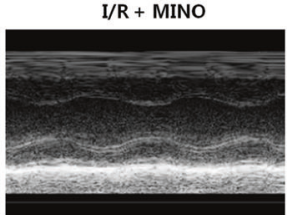

b
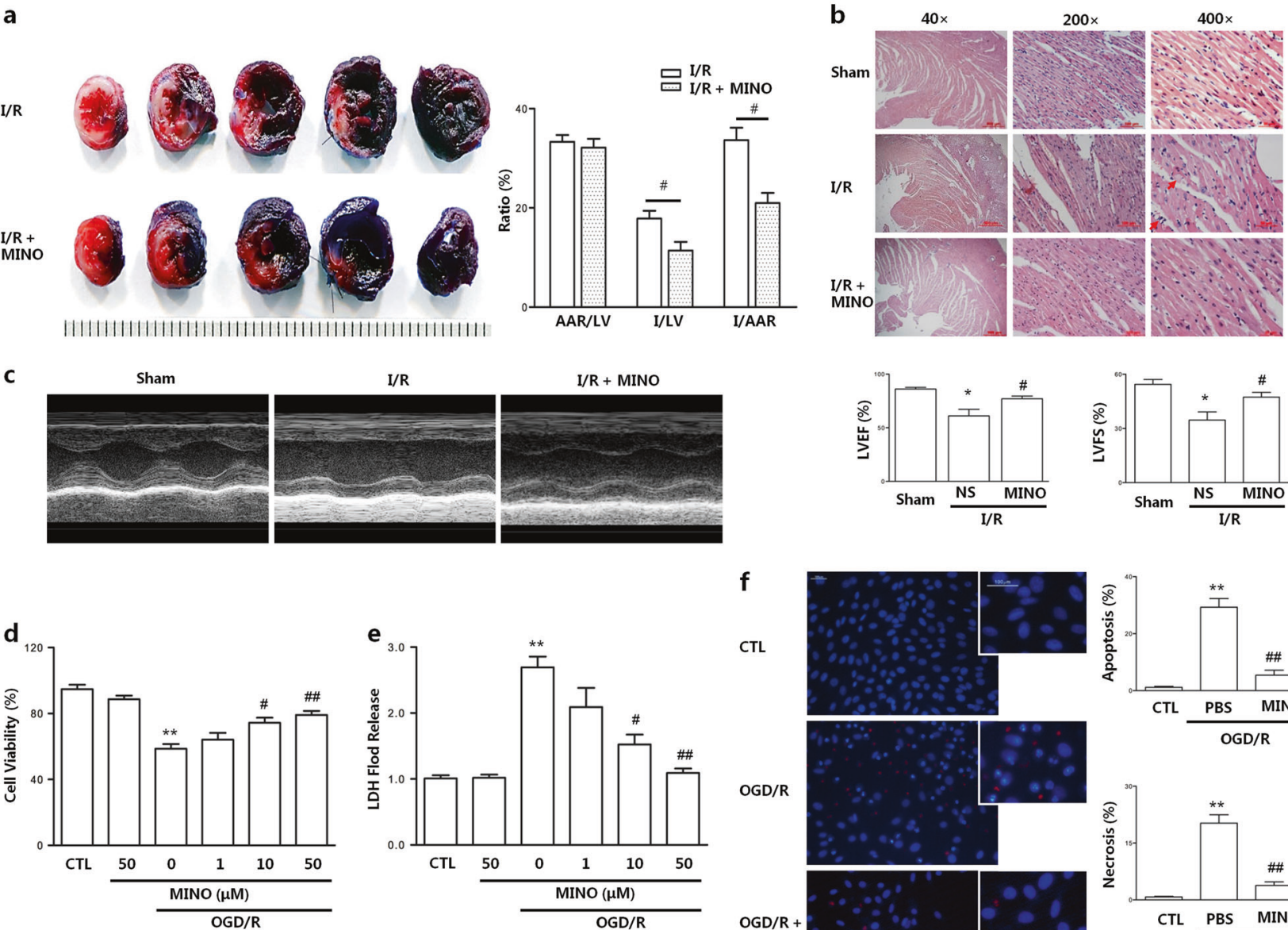

$200 \times$

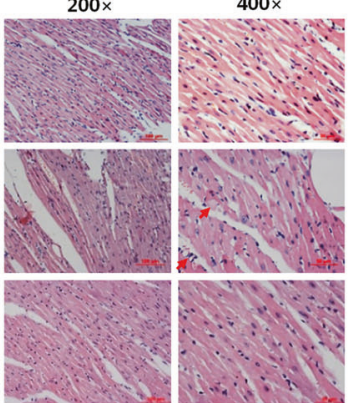

f

CTL
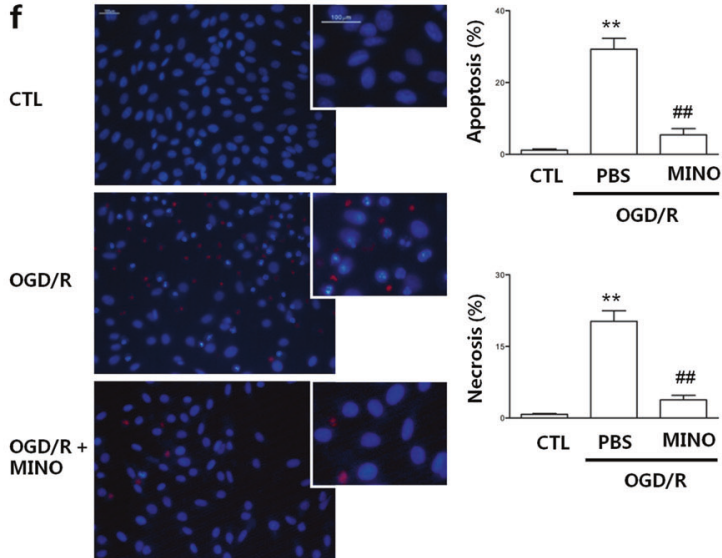

Fig. 1 Minocycline protects cardiomyocytes against I/R-induced injury in vivo and OGD/R-mediated I/R injury in vitro. The infarct size, myocardial histology and cardiac functions were assessed in rats after I ( $1 \mathrm{~h}) / \mathrm{R}(48 \mathrm{~h})$. a Infarct images obtained by TTC staining. Non-ischemic myocardium was stained blue, the ischemic area was stained red, and the infarcted area was stained pale. $\mathbf{b}$ Histochemical sections of the left ventricle were stained with hematoxylin and eosin. Scale bar $=500,100$, and $50 \mu \mathrm{m}$; c Cardiac function measurement was performed by echocardiography. Cell viability, lactate dehydrogenase (LDH) release, apoptosis, and necrosis were assessed in H9c2 cardiomyocytes at OGD $(6 \mathrm{~h}) / \mathrm{R}(6 \mathrm{~h})$ time. $\mathbf{d}$ Cell viability was analyzed by MTS assay. e Cell damage was determined by LDH release assay. $\mathbf{f}$ Representative figure of apoptosis (Hoechst staining showed bright blue nuclear condensation and fragmentation) and necrosis (PI-positive) as detected by Hoechst3324-propodium iodide double staining and the statistical graphs of apoptosis and necrosis. Scale bar $=100 \mu \mathrm{m}$; LVEF; left ventricle ejection fraction, LVFS; left ventricle fractional shortening. Values represent the mean \pm SEM. $n=6-8$ rats per group or 6 examples of cells; ${ }^{*} P<$ $0.05,{ }^{* *} P<0.01$ compared with the sham or CTL group; ${ }^{\#} P<0.05,{ }^{\# \#} P<0.01$ versus the I/R rats model group or OGD/R. CTL, normal control; $\mathrm{OGD} / \mathrm{R}$, oxygen glucose deprivation /reoxygenation 

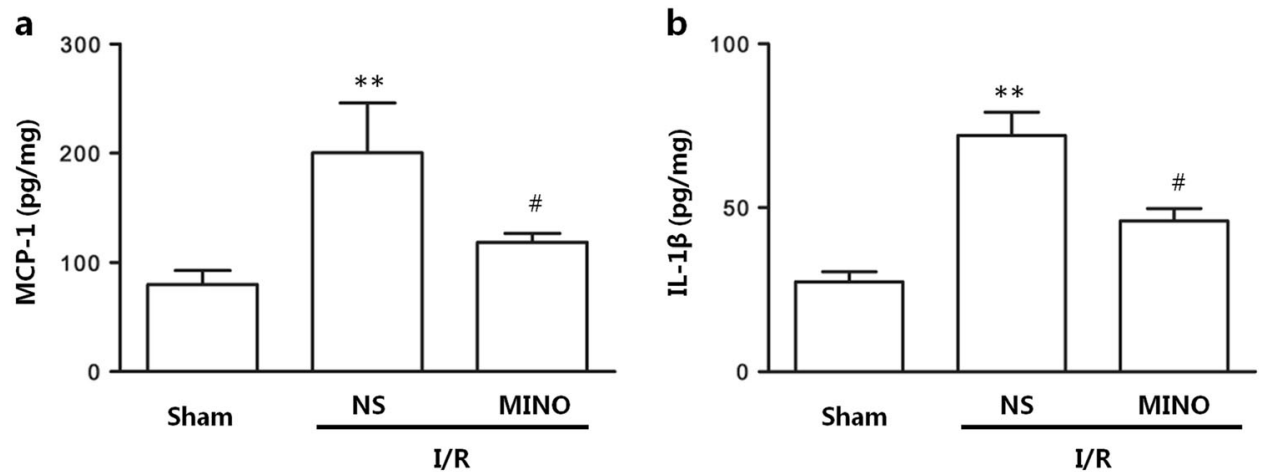

Fig. 2 Minocycline inhibits a MCP-1 and $\mathbf{b}$ IL- $1 \beta$ secretion induced by I/R injury in vivo. The protein levels of MCP-1 and IL-1 $\beta$ in the ischemic myocardium were assessed by a Bio-Plex Pro ${ }^{\text {TM }}$ kit. The values represent the mean \pm SEM, $n=6$ rats per group; ${ }^{*} P<0.05{ }^{* * *} P<0.01$ compared with the Sham group; ${ }^{\#} P<0.05$, ${ }^{\#} P<0.01$ versus the I/R model group. MCP-1, monocyte chemoattractant protein-1

a
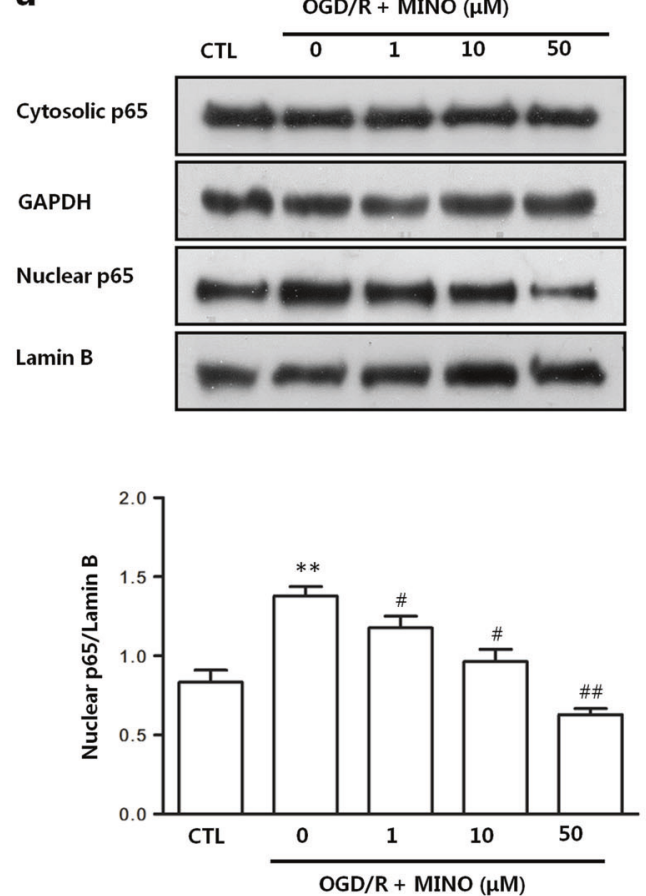

b
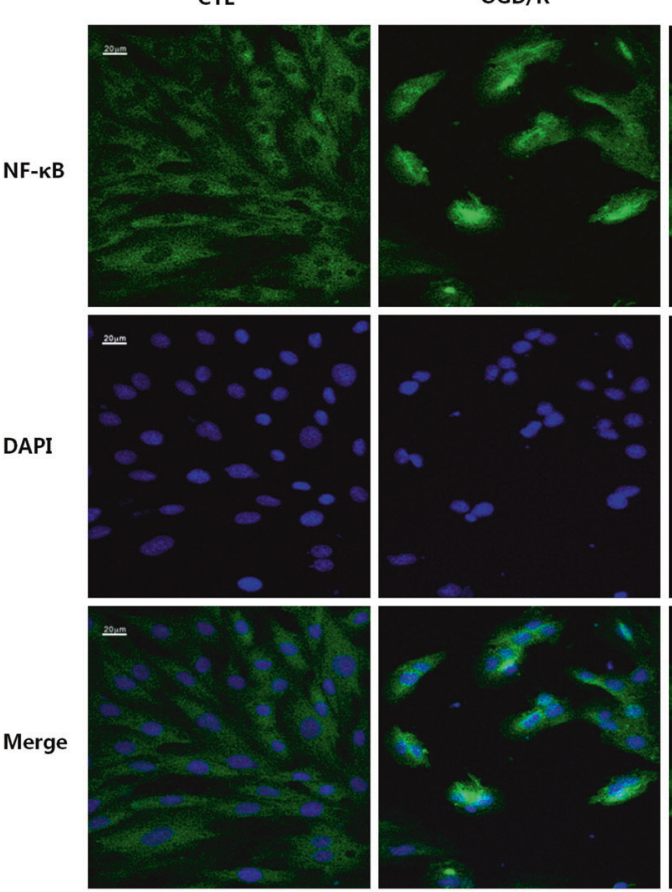

OGD/R + MINO

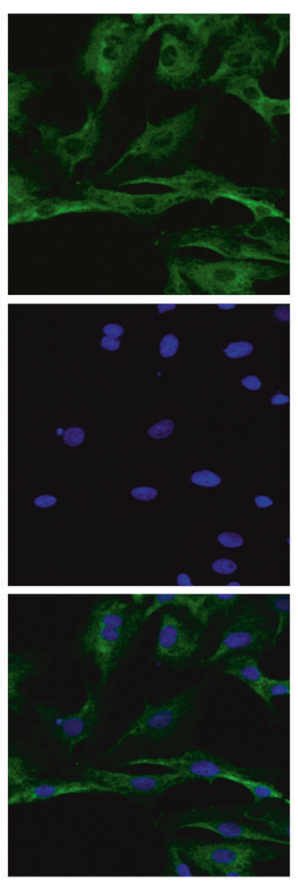

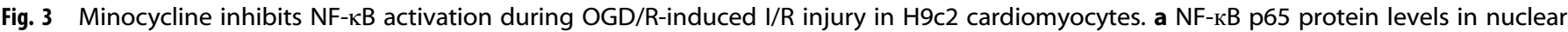
and cytosolic extracts were assayed by Western blot, and densitometry measurements of the nuclear p65 protein are normalized to Lamin $B$ or GAPDH. b Representative figures of NF-kB p65 nuclear translocation imaged by laser confocal immunofluorescent microscopy. Scale bar $=$ $20 \mu \mathrm{m}$. Quantitative data are presented as the mean \pm SEM. The results are representative of three independent experiments; ${ }^{*} P<0.05,{ }^{* *} P<$ 0.01 versus $C T L ;{ }^{\#} P<0.05$, ${ }^{\#} P<0.01$ versus the $O G D / R$ model

evaluated via transthoracic echocardiography (Fig. 1c), and LVEF output was significantly lower in the I/R model group than in the sham control group, while rats treated with minocycline showed increased LVEF $(61.05 \pm 16.32 \%$ vs $86.00 \pm 2.86 \% ; \quad P<0.05)$. Furthermore, I/R injury resulted in significantly reduced LVFS compared to the sham control group, while treatment with minocycline improved LVFS (34.60 $\pm 11.91 \%$ vs $54.43 \pm 5.42 \%$; $P<$ $0.01)$, although not to the level observed in the sham control group. These data suggested that minocycline plays a protective role following myocardial I/R infarction.

To further confirm the role of minocycline in OGD/R-mediated I/R injury, cell viability and the extent of cell damage were evaluated upon minocycline treatment of H9c2 cells. The MTS assay showed that OGD/R significantly reduced the cell viability of $\mathrm{H} 9 \mathrm{c} 2$ cells; however, administration of minocycline following
$\mathrm{OGD} / \mathrm{R}$ induction increased cell viability in a minocycline dosedependent manner $(P<0.01$ each compared to OGD/R model; Fig. 1d). Conversely, cell damage as evaluated by the $L D H$ release assay showed a steady reduction in LDH levels, indicating reduced cell damage with increasing minocycline dose following OGD/R-induced $\mathrm{I} / \mathrm{R}$ injury $(P<0.01$ each compared to OGD/R model; Fig. 1e). Furthermore, the cell apoptosis assay showed that OGD/R treatment increased the proportion of apoptotic and necrotic cells, as assessed by Hoescht3324 and PI staining, respectively, compared to control cells. However, treatment with $50 \mu \mathrm{M}$ minocycline abrogated OGD/R-mediated I/R injury, suggesting a cardioprotective role $(P<0.01$ compared to OGD/R model; Fig. 1f). 
a

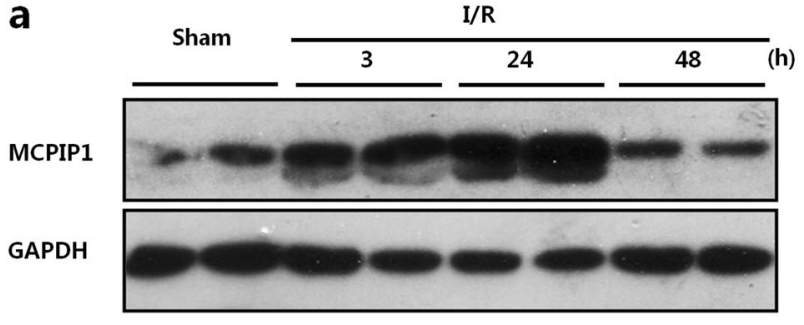

b

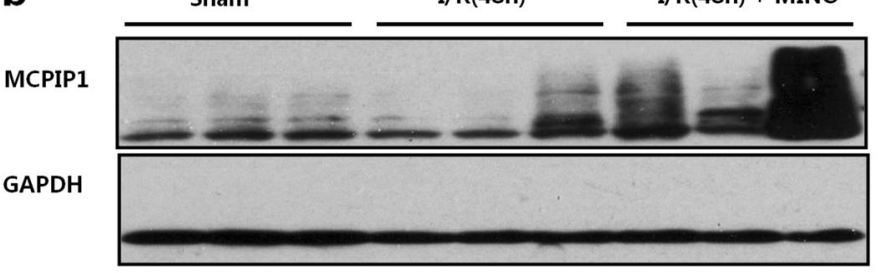

C

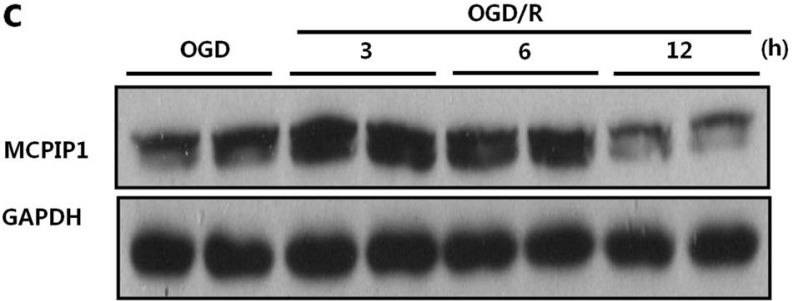

d

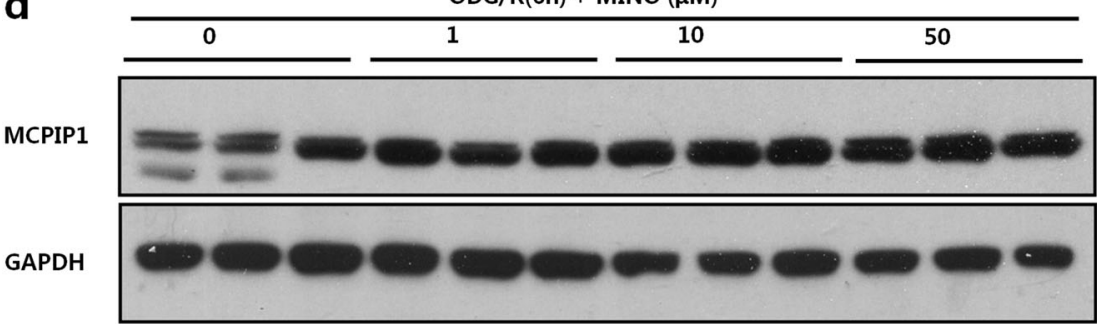

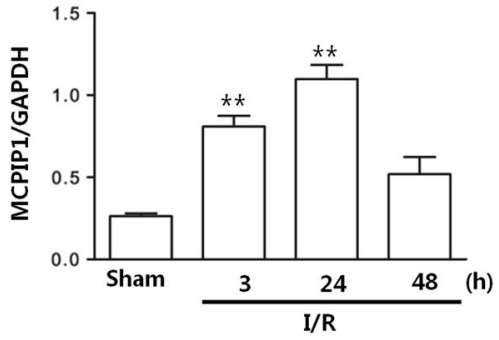
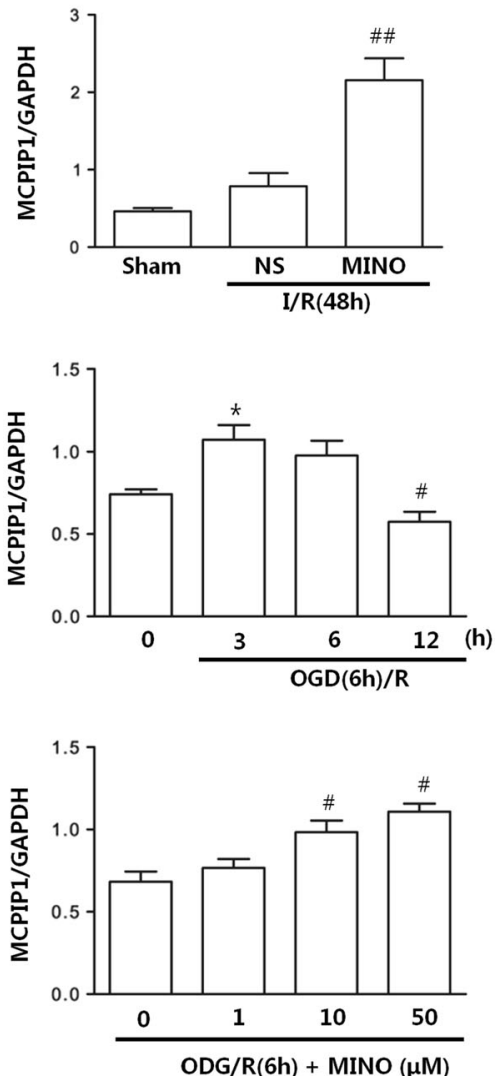

Fig. 4 Minocycline induces MCPIP1 expression during I/R-induced injury in vivo and OGD/R-induced I/R injury in vitro. a MCPIP1 protein levels in ischemic myocardial tissue were measured by Western blot at I $(1 \mathrm{~h}) / \mathrm{R}(3 \mathrm{~h}), \mathrm{I}(\mathrm{h}) / \mathrm{R}(24 \mathrm{~h})$, and I $(1 \mathrm{~h}) / \mathrm{R}(48 \mathrm{~h})$. b MCPIP1 protein levels with and without minocycline treatment were measured by Western blot after I (1 h)/R (48). c MCPIP1 protein levels in H9c2 were measured by Western blot at different oxygen glucose deprivation (OGD)-reoxygenation times (OGD6 h/R3, 6, 12 h). d MCPIP1 protein levels with minocycline treatment in $\mathrm{H} 9 \mathrm{c} 2$ cells subjected to OGD/R were measured in a concentration-dependent manner by Western blot. Quantitative data are presented as the mean \pm SEM. $n=6$ rats per group or 6 examples of cells; ${ }^{*} P<0.05,{ }^{* *} P<0.01$ versus Sham or $C T L ;{ }^{\#} P<0.05,{ }^{\# \#} P<0.01$ versus I/R model or OGD/R model. MCPIP1, monocyte chemotactic protein-induced protein-1

Minocycline inhibits monocyte chemoattractant protein-1 (MCP-1) and IL- $1 \beta$ secretion induced by $\mathrm{I} / \mathrm{R}$ injury in vivo

In addition, $\mathrm{I} / \mathrm{R}$ injury resulted in significantly increased levels of MCP-1 and IL-1 $\beta$ compared to sham treatment $(79.93 \pm 34.24$ vs $200.48 \pm 102.04 \mathrm{pg} / \mathrm{mg} ; \quad 26.91 \pm 8.03$ vs $62.20 \pm 21.33 \mathrm{pg} / \mathrm{mg}$; respectively; $P<0.01$, Fig. $2 \mathrm{a}, \mathrm{b})$. However, these increases were inhibited by minocycline treatment, with reduction of MCP-1 levels to $118.37 \pm 18.22 \mathrm{pg} / \mathrm{mg}$ and IL-1 $\beta$ levels to $41.21 \pm 9.14 \mathrm{pg} /$ mg (both $P<0.05$ compared to the I/R model, Fig. 2a, b) in AAR.

Minocycline inhibits NF-KB activation during OGD/R-mediated I/R injury in $\mathrm{H} 9 \mathrm{c} 2$ cardiomyocytes

Activation of the NF-KB signaling pathway leads to production of pro-inflammatory cytokines such as IL-6, TNF- $a$, and MCP-1 [24, 25], and a previous study showed that NF-KB subunit p65 promotes the inflammatory response in liver I/R injury [26]. We therefore examined whether minocycline treatment influenced NF-KB activation in this setting. Western blot and laser confocal immunofluorescence microscopy results showed that nuclear translocation of NF-KB p-65 induced by OGD/Rinduced I/R injury was weakened by minocycline treatment in a dose-dependent manner (Fig. 3a, b; $P<0.01$ compared to the OGD/R model).

Minocycline induces MCPIP1 expression during I/R injury in vivo and OGD/R-mediated I/R injury in vitro

A recent study showed that MCPIP1 can mediate minocyclineinduced protection in cerebral I/R [14]. To determine whether such a mechanism is at play in myocardial $I / R$, we first evaluated whether treatment with minocycline induced MCPIP1 expression in AAR areas. Following $1 \mathrm{~h}$ of infarction, MCPIP1 protein expression levels increased over $24 \mathrm{~h}$ of reperfusion $(P<0.01$ each compared to sham) before decreasing at $48 \mathrm{~h}$ post-reperfusion (Fig. 4a; $P>0.05$ compared to sham). However, treatment with minocycline significantly induced MCPIP1 expression following $1 \mathrm{~h}$ of infarction and $48 \mathrm{~h}$ of reperfusion (Fig. $4 \mathrm{~b} ; P<0.01$ ). Next, we 

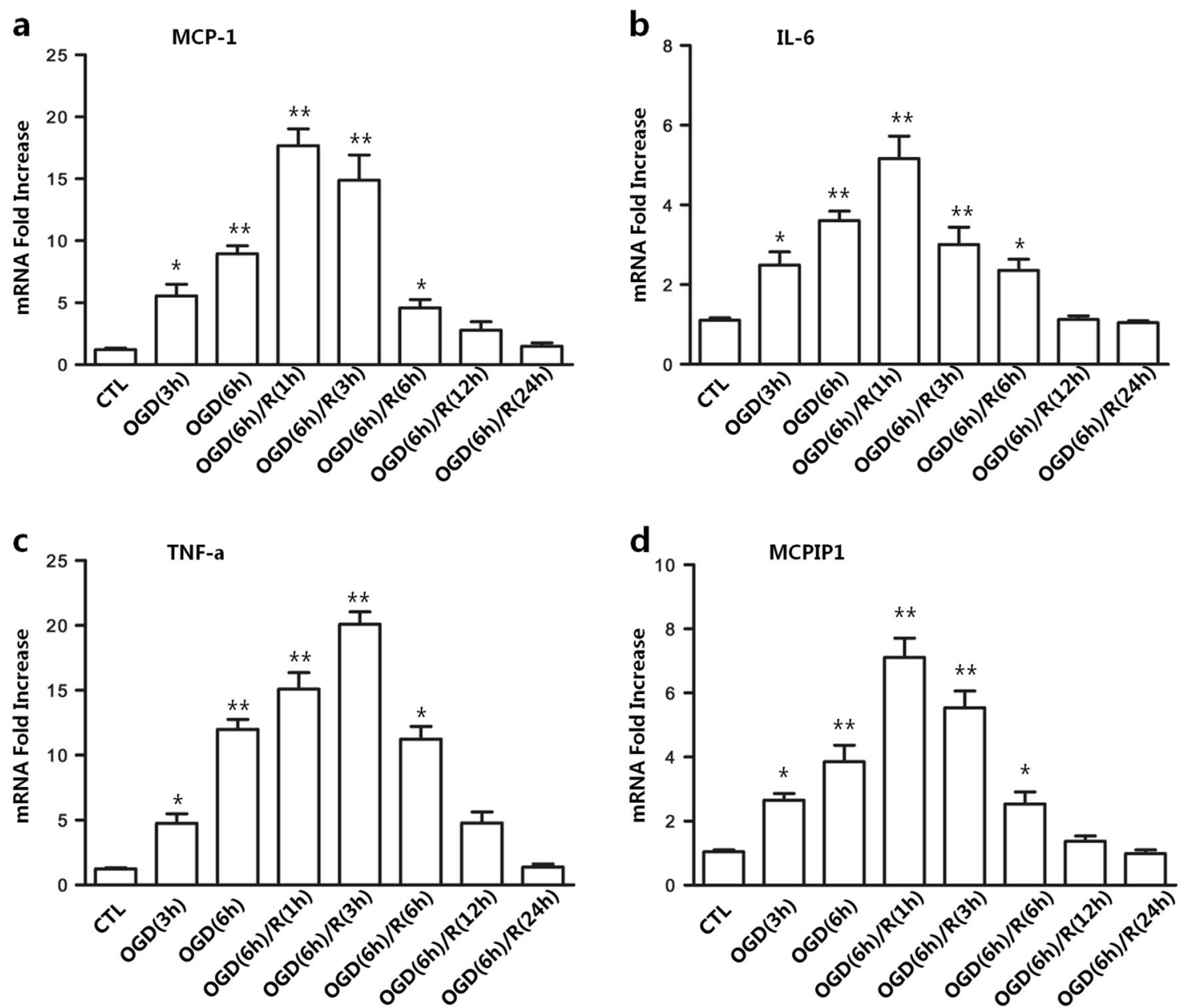

Fig. 5 MCPIP1 and pro-inflammatory cytokines expression are induced during OGD-R-induced I/R injury in H9c cells. The relative mRNA levels of a MCP-1, b IL-6, and c TNF- $\alpha$ and MCPIP1 $\mathbf{d}$ in H9c2 cells were determined by qPCR at different oxygen glucose deprivation times (OGD 3, 6 h) and OGD-reoxygenation times (R1, 3, 6, 12, $24 \mathrm{~h})$. The relative levels were normalized to GAPDH. Quantitative data are presented as the mean \pm SEM. $n=6 ;{ }^{*} P<0.05,{ }^{* *} P<0.01$ versus $C T L ;{ }^{\#} P<0.05,{ }^{\# \#} P<0.01$ versus OGD/R model

evaluated MCPIP1 expression following OGD/R-induced I/R injury in $\mathrm{H} 9 \mathrm{c} 2$ cells. As depicted in Fig. 4c, d, MCPIP1 protein levels increased in a dose-dependent manner with minocycline treatment. These data implicate MCPIP1 in minocycline-induced cardioprotection during I/R injury in vivo and in vitro.

MCPIP1 and pro-inflammatory cytokine expression are induced during OGD/R-mediated I/R injury in $\mathrm{H} 9 \mathrm{c} 2$ cells

Inflammation is a major contributor to cardiac I/R injury and is mainly mediated by pro-inflammatory cytokines. To establish I/R conditions in vitro, $\mathrm{H} 9 \mathrm{c} 2$ cells were subjected to OGD followed by reoxygenation $(\mathrm{OGD} / \mathrm{R})$, and levels of pro-inflammatory cytokines were evaluated. The gene expression levels of $M C P-1, I L-6$, and TNF- $a$ steadily increased over $6 \mathrm{~h}$ following OGD treatment compared to control cells (Fig. 5a-c; $P<0.01$ ). Moreover, significant induction of these pro-inflammatory cytokines was observed upon reoxygenation (OGD/R), with peak levels at $1 \mathrm{~h}$ for $M C P-1$ and IL-6 and $3 \mathrm{~h}$ for TNF- $a$, before steadily decreasing over $24 \mathrm{~h}(P<0.01)$. We next examined the impact of OGD/R-induced I/ $\mathrm{R}$ injury on MCPIP1 expression, and similar to our observations for the pro-inflammatory cytokines, MCPIP1 transcript (Fig. 5d) levels increased over time with OGD and were significantly upregulated upon reoxygenation $(P<0.01)$, before decreasing over $24 \mathrm{~h}$ thereafter. These results indicate that in vitro OGD/R-induced I/R conditions can induce the expression of MCPIP1 and proinflammatory cytokines in $\mathrm{H} 9 \mathrm{c} 2$ cells.
MCPIP1 knockdown promotes the expression of MCP-1 and IL-6 and reduces cell viability during OGD/R-mediated I/R injury in vitro We next sought to explore whether a relationship exists between MCPIP1 and pro-inflammatory cytokines during OGD/R-induced I/ R. RNAi-mediated knockdown of MCPIP1 was conducted in $\mathrm{H} 9 \mathrm{c} 2$ cells followed by OGD/R treatment, and the levels of MCP-1, IL-6, and TNF-a were determined. Transfection with three siRNA oligonucleotides targeting various locations of the MCPIP1 gene was performed. As shown in Supplementary Fig. S1, all three siMCPIP1 transfections had great efficiency. The highest levels of cleaved caspase-3 were achieved with siMCPIP1-1, which was therefore used for subsequent experiments (Supplementary Fig. S2). In siMCPIP1 transfectants, $M C P-1$ and $I L-6$ increased compared to non-transfected and siControl-transfected cells at $1 \mathrm{~h}$ post-reoxygenation before decreasing after $6 \mathrm{~h}$, although still significantly higher (Fig. 6a, b).TNF-a levels were reduced in all groups at $6 \mathrm{~h}$ of reoxygenation compared to $3 \mathrm{~h}$, but no differences were observed in the expression levels among the three groups at either time point (Fig. 6c).

We also evaluated the impact of MCPIP1 knockdown on cell viability. Phase contrast imaging of $\mathrm{H} 9 \mathrm{c} 2$ morphology showed a low refractive index and fuzzy structure (greater number of autophagic vacuoles) in siMCPIP1 transfectants compared to siControl transfectants (Fig. 6d). Data from MTS assay revealed that MCPIP1 knockdown significantly reduced cell viability compared to the siControl group (Fig. 6e; $P<0.05$ ). 

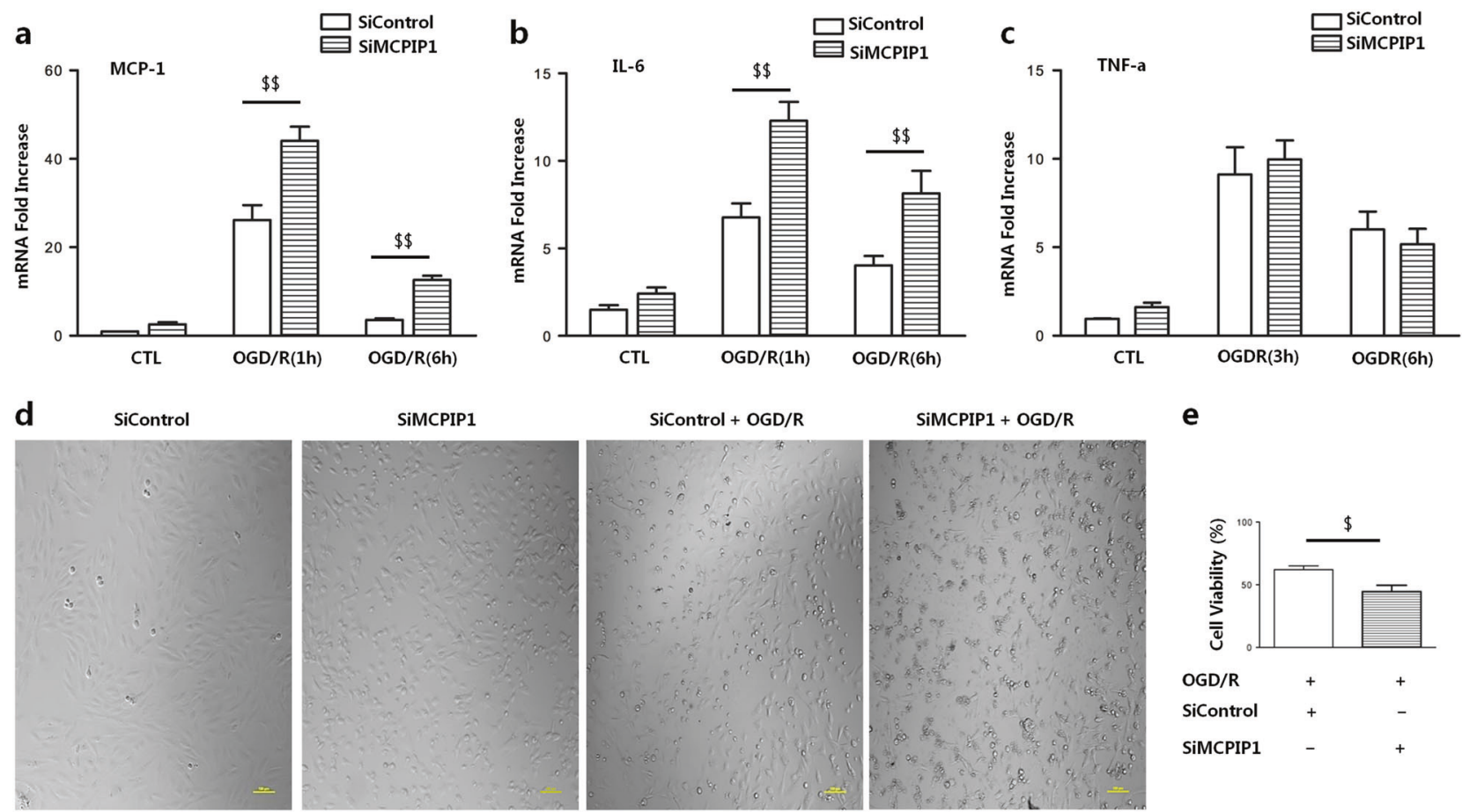

SiMCPIP1 + OGD/R

e

Fig. 6 MCPIP1 knockdown promotes the expression of MCP-1 and IL- 6 and reduces cell viability during OGD/R-induced I/R injury in vitro. The relative mRNA levels of pro-inflammatory cytokines a MCP-1, b IL-6, and c TNF- $\alpha$ were measured by qPCR after knockdown of MCPIP1-1 in $\mathrm{H} 9 \mathrm{c} 2$ cells. d Representative images of $\mathrm{H} 9 \mathrm{c} 2$ cell morphology were obtained under a light microscope. e The viability of H9c2 cells was determined by MTS assay after siMCPIP1-1 transfection. Scale bar $=100 \mu \mathrm{m}$. Quantitative data are presented as the mean \pm SEM. $n=6$; ${ }^{*} P<$ $0.05,{ }^{* *} P<0.01$ versus $C T L ;{ }^{\#} P<0.05,{ }^{\# \#} P<0.01$ versus OGD/R model; ${ }^{\$} P<0.05,{ }^{\$ \$} P<0.01$ versus siControl. siControl, negative control siRNA; SiMCPIP, MCPIP1 SiRNA

These data suggested a cardioprotective role of MCPIP1 in OGD/R-induced $I / R$ injury and that this protective effect seems to be achieved by inhibiting MCP-1 and IL- 6 but not TNF-a.

Minocycline protects $\mathrm{H} 9 \mathrm{c} 2$ cardiomyocytes against OGD/Rinduced I/R injury in $\mathrm{H} 9 \mathrm{c} 2$ cardiomyocytes via MCPIP

As our data implicated MCPIP1 in minocycline-induced cardioprotection during I/R injury (Fig. 6), we sought to confirm this relationship. The MTS assay showed that OGD/R treatment reduced cell viability, which was reversed with minocycline treatment; however, siRNA-mediated inhibition of MCPIP1 reduced cell viability regardless of minocycline treatment (Fig. 7a). Moreover, OGD/R treatment increased transcript levels of MCP-1 and IL-6 ( $P<0.05$ each compared to untreated controls), which were inhibited by minocycline treatment. However, knockdown of MCPIP1 significantly increased MCP-1 and IL-6 levels irrespective of minocycline treatment (Fig. 7b, $c ; P<0.05$ each compared to siControl). These data provide direct evidence that the cardioprotective effects of minocycline in OGD/R-induced I/R injury are mediated by MCPIP1.

\section{DISCUSSION}

Timely reperfusion following ischemia has greatly contributed to reducing mortality rates associated with acute myocardial infarction; however, these strategies have unexpectedly contributed to higher incidences of chronic heart failure [1]. Consequently, research studies are currently aimed at reducing infarct size and reperfusion injury stage. Over the past two decades, studies have shown that in addition to its antibacterial activity, minocycline exhibits anti-inflammatory functions and has a neuroprotective role in cerebral ischemia [27-29]. Here, we showed that minocycline induced myocardial protection against
I/R injury in both in vivo and in vitro models. Minocycline has previously been shown to protect OGD/R-injured vascular endothelial cells by inducing autophagy [22]. In the present study, we demonstrated that minocycline protected H9c2 cardiomyocytes against OGD/R-induced I/R injury, increasing cell viability and reducing apoptosis and necrosis. Furthermore, our in vivo observations were extended in a rat model of I/R injury, in which 3-day minocycline treatment significantly reduced the infarct size. These results were consistent with those of previous studies $[12,13,23]$. Importantly, improved cardiac function was observed in minocycline-treated animals, with LVEF function restored to similar levels as control animals, in addition to improved LVFS function.

Initial myocardial ischemic injury triggers oxidative stress, resulting in production of reactive oxygen species (ROS) from various sources, such as mitochondria and endothelial nitric oxide synthase (eNOS) [30]. Consequently, in response to ROS, proinflammatory cytokines are released, and reperfusion injury is associated with an inflammatory cascade, which further contributes to the myocardial dysfunction observed during I/R due to sustained inflammatory responses. Therefore, it is feasible that blocking the production of these pro-inflammatory cytokines would be an important strategy to protect against myocardial I/R injury [31-33]. Our study confirmed that MCP-1, IL-6, and TNF-a were induced following $O G D / R$, which mimics ischemia, and these pro-inflammatory cytokines were produced within the initial stages of reperfusion. Subsequently, expression of these cytokines activates the NF-KB signaling pathway, which is a mediator of I/R injury. The overall outcome of NF-KB activation is an inflammatory response characterized by enhanced expression of pro-inflammatory cytokines such as TNF-a, IL-1, IL-6, and IL-8, chemokines such as MCP-1 and macrophage inflammatory protein-1a (MIP-1a), and inducible enzymes that produce 
a

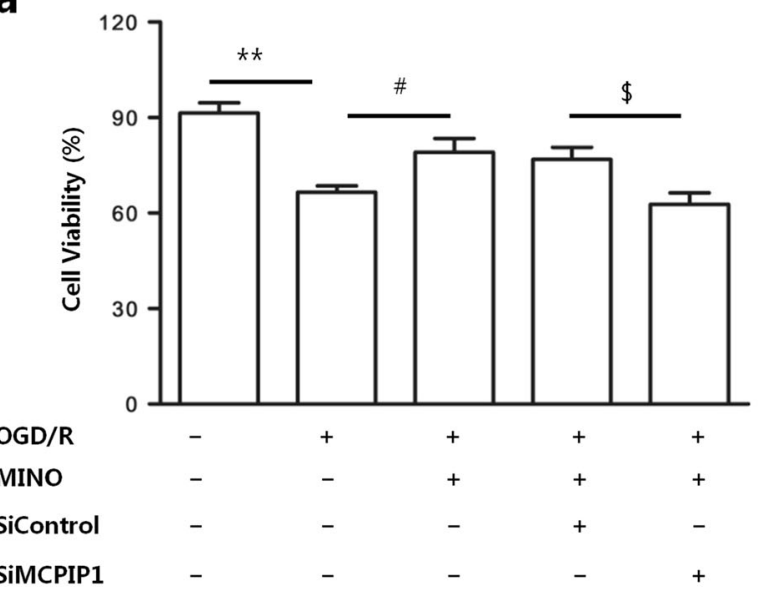

b

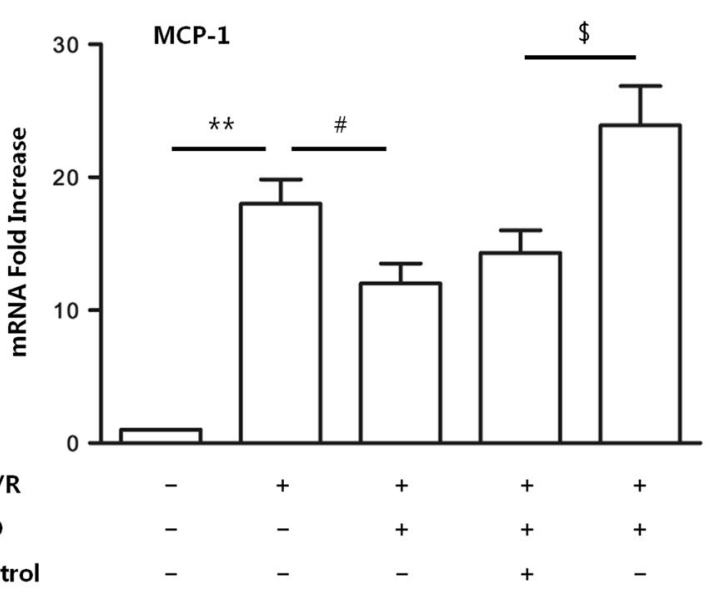

C

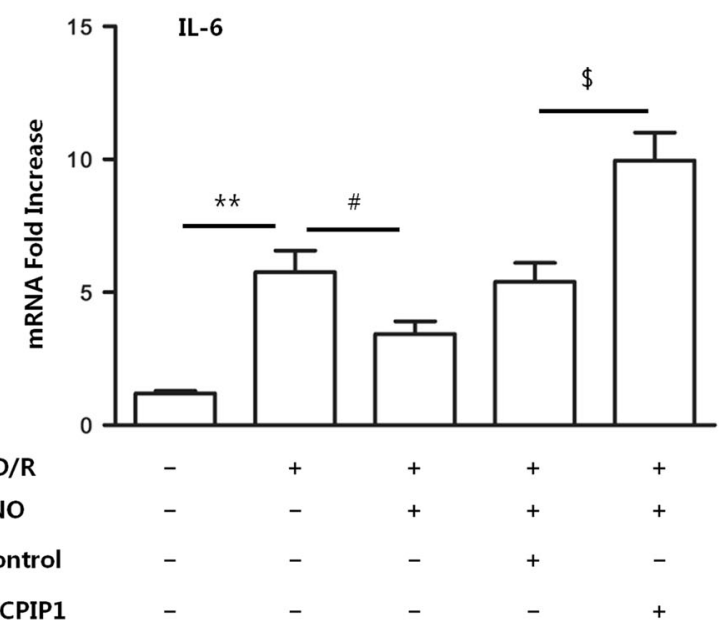

Fig. 7 Minocycline protects $\mathrm{H} 9 \mathrm{c} 2$ cardiomyocytes against OGD/R-induced I/R injury in H9c2 cardiomyocytes via MCPIP1. a The cell viability was determined by MTS assay, and the relative mRNA levels of the pro-inflammatory cytokines $\mathbf{b}$ MCP-1, and c IL- 6 were measured by qPCR in $\mathrm{H} 9 \mathrm{c} 2$ cells subjected to OGD/R injury pretreated with minocycline after knockdown of MCPIP1 by RNAi. Quantitative data are presented as the mean \pm SEM. $n=6 ;{ }^{*} P<0.05,{ }^{* *} P<0.01$ versus CTL; ${ }^{\#} P<0.05,{ }^{\# \#} P<0.01$ versus OGD/R model; ${ }^{\$} P<0.05,{ }^{\$ \$} P<0.01$ versus siControl +0 OGD/R + MINO. siControl, negative control siRNA; siMCPIP, MCPIP1 siRNA

secondary inflammatory mediators such as COX-2 and induced NO synthase (iNOS). This is a vicious cycle that expands the initial inflammatory response [34], which is an important mechanism of $\mathrm{I} / \mathrm{R}$ injury [35]. Reducing the excessive activation of NF-KB could inhibit the inflammatory response and improve I/R damage [31-33]. Although it has been reported that minocycline inhibits inflammatory responses such as macrophage and macrophagelike cell activation [36, 37] and pro-inflammatory cytokine generation stimulated by lipopolysaccharide (LPS) [10, 38], whether minocycline reduces inflammation after myocardial I/R injury and the molecular mechanisms that contribute to the alleviation of inflammation after myocardial $\mathrm{I} / \mathrm{R}$ are not well understood. In the present study, treatment with minocycline inhibited the expression of MCP-1, IL- $1 \beta$, and IL- 6 in vivo and in vitro and, more importantly, inhibited NF-KB p65 nuclear translocation, indicating activation of the NF-KB signaling pathway.

We further determined that minocycline mediated its cardioprotective functions by inducing MCPIP1. Previous research has shown that MCPIP1 suppresses the inflammatory response through multiple mechanisms, including inhibiting NF-KB activation upon LPS stimulation in macrophages and by targeting TNF receptor-associated factors (TRAFs) as a deubiquitinase $[7,8,39$, 40]; moreover, MCPIP1 is an RNase and thus destabilizes a set of cytokine-encoding mRNAs such as IL-6, IL- $1 \mathrm{~b}$, and IL-12p40 $[41,42]$, which may lead to reduced expression of some proinflammatory mediators. Herein, we observed a significant increase in MCPIP1 expression in response to minocycline treatment both in vivo and in vitro. Furthermore, siRNAmediated inhibition of MCPIP1 significantly increased the gene expression of MCP-1 and IL-6 in H9c2 cells following OGD/R treatment and abrogated the minocycline-induced cardioprotective effects, as indicated by reduced cell viability and increased production of MCP-1 and IL-6. While we did not directly evaluate the impact of minocycline treatment on NF-KB pathway activation in siMCPIP1 transfectants, previous studies have shown that minocycline inhibits NF-KB activation in the ischemic brain and that this effect is abolished by MCPIP1 deletion [14]. Consistently, we found that minocycline inhibits NF-KB pathway activation in ischemic myocardiocytes. Further studies are needed to demonstrate whether MCPIP1 is involved in the effect of minocycline on NF-KB in myocardiocytes. Nonetheless, our data indicate that the loss of MCPIP1 is a key event that cannot be bypassed by minocycline administration.

In conclusion, we found that MCPIP1 is involved in the pathological process of myocardial $I / R$ and negatively regulates the production of inflammatory factors induced by myocardial I/R. These results imply that MCPIP1 may be a 
potential therapeutic target for limiting the severity of myocardial I/R injury.

This study also builds upon a body of evidence showing the protective effects of minocycline in myocardial $I / R$ and provides proof-of-concept that minocycline, as part of its antiinflammatory function, promotes the expression of the endogenous protein MCPIP1 in I/R. The novel mechanism of minocyclineMCPIP1-NF-KB reinforces the notion that minocycline is an agent that may effectively target myocardial I/R injury, especially during the reperfusion stage.

\section{ACKNOWLEDGEMENTS}

This study was supported by the National Natural Science Foundation of China (No: 81573433 to Jian-dong Luo), the Guangdong Provincial Science and Technology Department Program Grant (No: 2012B031800235 to Quan Yi) and the Guangzhou City Bureau of Education General Program Grant (No: $2012 \mathrm{C} 125$ to Quan Yi). We thank Xiao-qian Wu, Gen-sui Zhang, Hai-ning Zhang, Qin Xue, Ning Hou, Yun Liu and Xiao-yan Dai for valuable guidance in experiments and at every stage of the writing.

\section{AUTHOR CONTRIBUTION}

Q.Y. and J.-d.L. designed the research; Q.Y., F.-h.T., J.-a.T., and X.-h.C. performed the research; Q.X. and Y.-h.L. analyzed the data; G.-p.Z. assisted with laser confocal microscopy; and Q.Y. wrote the paper.

\section{ADDITIONAL INFORMATION}

The online version of this article (https://doi.org/10.1038/s41401-019-0214-z) contains supplementary material, which is available to authorized users.

\section{Competing interests: None}

\section{REFERENCES}

1. Ibanez B, Heusch G, Ovize M, Van de Werf F. Evolving therapies for myocardial ischemia/reperfusion injury. J Am Coll Cardiol. 2015;65:1454-71.

2. Sharma HS, Das DK. Role of cytokines in myocardial ischemia and reperfusion. Mediat Inflamm. 1997;6:175-83.

3. Marchant DJ, et al. Inflammation in myocardial diseases. Circ Res. 2012;110:126-44.

4. Sanchis-Gomar F, Perez-Quilis C, Leischik R, Lucia A. Epidemiology of coronary heart disease and acute coronary syndrome. Ann Transl Med. 2016;4:256.

5. Zhou $L$, et al. Monocyte chemoattractant protein-1 induces a novel transcription factor that causes cardiac myocyte apoptosis and ventricular dysfunction. Circ Res. 2006;98:1177-85.

6. Fu M, Blackshear PJ. RNA-binding proteins in immune regulation: a focus on CCCH zinc finger proteins. Nat Rev Immunol. 2017;17:130-43.

7. Liang J, et al. MCP-induced protein 1 deubiquitinates TRAF proteins and negatively regulates JNK and NF-kB signaling. J Exp Med. 2010;207:2959-73.

8. Liang J, et al. A novel $\mathrm{CCCH}$-zinc finger protein family regulates proinflammatory activation of macrophages. J Biol Chem. 2008;283:6337-46.

9. Niu J, Jin Z, Kim H, Kolattukudy PE. MCP-1-induced protein attenuates post-infarct cardiac remodeling and dysfunction through mitigating NF-kB activation and suppressing inflammation-associated microRNA expression. Basic Res Cardiol. 2015;110:26.

10. Tai K, Iwasaki H, Ikegaya S, Ueda T. Minocycline modulates cytokine and chemokine production in lipopolysaccharide-stimulated THP-1 monocytic cells by inhibiting IKB kinase $\alpha / \beta$ phosphorylation. Transl Res. 2013;161:99-109.

11. Garrido-Mesa N, Zarzuelo A, Galvez J. Minocycline: far beyond an antibiotic. Br J Pharmacol. 2013;169:337-52.

12. Scarabelli TM, et al. Minocycline inhibits caspase activation and reactivation, increases the ratio of XIAP to smac/DIABLO, and reduces the mitochondrial leakage of cytochrome C and smac/DIABLO. J Am Coll Cardiol. 2004;43:865-74.

13. Romero-Perez $D$, et al. Cardiac uptake of minocycline and mechanisms for in vivo cardioprotection. J Am Coll Cardiol. 2008;52:1086-94.

14. Jin Z, Liang J, Wang J, Kolattukudy PE. MCP-induced protein 1 mediates the minocycline-induced neuroprotection against cerebral ischemia/reperfusion injury in vitro and in vivo. J Neuroinflamm. 2015;12:39.
15. Wu X, et al. Induction of autophagy contributes to the myocardial protection of valsartan against ischemiareperfusion injury. Mol Med Rep. 2013;8:1824-30.

16. Xiao $Q$, et al. Impaired sonic hedgehog pathway contributes to cardiac dysfunction in type 1 diabetic mice with myocardial infarction. Cardiovasc Res. 2012;95:507-16.

17. Chen K, et al. Quercetin prevents in vivo and in vitro myocardial hypertrophy through the proteasome-GSK-3 pathway. Cardiovasc Drugs Ther. 2018;32:5-21.

18. Bohl S, et al. Refined approach for quantification of in vivo ischemia-reperfusion injury in the mouse heart. Am J Physiol Heart Circ Physiol. 2009;297:H2054-8.

19. Zhang $\mathrm{HN}$, et al. Endogenous glucocorticoids inhibit myocardial inflammation induced by lipopolysaccharide: involvement of regulation of histone deacetylation. J Cardiovasc Pharmacol. 2012;60:33-41.

20. Wu $\mathrm{X}$, et al. Impaired autophagy contributes to adverse cardiac remodeling in acute myocardial infarction. PLoS ONE. 2014;9:e112891.

21. He $\mathrm{YH}$, et al. A physiological concentration of glucocorticoid inhibits the proinflammatory cytokine-induced proliferation of adult rat cardiac fibroblasts: roles of extracellular signal-regulated kinase $1 / 2$ and nuclear factor-kB. Clin Exp Pharmacol Physiol. 2011;38:739-46.

22. Dong W, Xiao S, Cheng M, Ye X, Zheng G. Minocycline induces protective autophagy in vascular endothelial cells exposed to an in vitro model of ischemia/ reperfusion-induced injury. Biomed Rep. 2016;4:173-7.

23. $\mathrm{Hu} \mathrm{X}$, et al. Minocycline protects against myocardial ischemia and reperfusion injury by inhibiting high mobility group box 1 protein in rats. Eur J Pharmacol. 2010;638:84-9.

24. Lawrence T. The nuclear factor NF-kB pathway in inflammation. Cold Spring Harb Perspect Biol. 2009;1:a001651.

25. Tak PP, Firestein GS. NF-kB: a key role in inflammatory diseases. J Clin Invest. 2001;107:7-11.

26. Ramachandran $S$, et al. Ischemia-reperfusion injury in rat steatotic liver is dependent on NFkB P65 activation. Transpl Immunol. 2012;26:201-6.

27. Fagan SC, Cronic LE, Hess DC. Minocycline development for acute ischemic stroke. Transl Stroke Res. 2011;2:202-8.

28. Yrjanheikki J, et al. A tetracycline derivative, minocycline, reduces inflammation and protects against focal cerebral ischemia with a wide therapeutic window. Proc Natl Acad Sci USA. 1999;96:13496-500.

29. Machado LS, et al. Delayed minocycline inhibits ischemia-activated matrix metalloproteinases 2 and 9 after experimental stroke. BMC Neurosci. 2006;7:56.

30. Di Lisa F, Bernardi P. Mitochondria and ischemia-reperfusion injury of the heart: fixing a hole. Cardiovasc Res. 2006;70:191-9.

31. Chung J. W. et al. Anti-inflammatory effects of (Z)-ligustilide through suppression of mitogen-activated protein kinases and nuclear factor-kappaB activation pathways. Arch. Pharm. Res.2012;35:723-32.

32. Kim JW, et al. Daidzein administration in vivo reduces myocardial injury in a rat ischemia/reperfusion model by inhibiting NF-kB activation. Life Sci. 2009;84:227-34.

33. $\mathrm{Ma} \mathrm{L}$, et al. Ginsenoside $\mathrm{Rb} 3$ protects cardiomyocytes against ischemiareperfusion injury via the inhibition of JNK-mediated NF-kB pathway: a mouse cardiomyocyte model. PLoS ONE. 2014;9:e103628.

34. Brigelius-Flohe R, Flohe L. Basic principles and emerging concepts in the redox control of transcription factors. Antioxid Redox Signal. 2011;15:2335-81.

35. Taqueti VR, Mitchell RN, Lichtman AH. Protecting the pump: controlling myocardial inflammatory responses. Annu Rev Physiol. 2006;68:67-95.

36. Song $\mathrm{X}$, et al. Minocycline protects melanocytes against $\mathrm{H} 2 \mathrm{O} 2$-induced cell death via JNK and p38 MAPK pathways. Int J Mol Med. 2008;22:9-16.

37. Nikodemova M, Duncan ID, Watters JJ. Minocycline exerts inhibitory effects on multiple mitogen-activated protein kinases and $\mathrm{I} \mathrm{KBa}$ degradation in a stimulusspecific manner in microglia. J Neurochem. 2006;96:314-23.

38. Huang WC, et al. Direct protection of cultured neurons from ischemia-like injury by minocycline. Anat Cell Biol. 2010;43:325-31.

39. Skalniak L, et al. Regulatory feedback loop between NF-kB and MCP-1-induced protein 1 RNase. Febs J. 2009;276:5892-905.

40. Niu J, Wang K, Graham S, Azfer A, Kolattukudy PE. MCP-1-induced protein attenuates endotoxin-induced myocardial dysfunction by suppressing cardiac NF-kB activation via inhibition of IkB kinase activation. J Mol Cell Cardiol. 2011;51:177-86.

41. Matsushita K, et al. Zc3h12a is an RNase essential for controlling immune responses by regulating mRNA decay. Nature. 2009;458:1185-90.

42. Mizgalska $D$, et al. Interleukin-1-inducible MCPIP protein has structural and functional properties of RNase and participates in degradation of IL-1 $\beta$ mRNA. FEBS J. 2009;276:7386-99. 